\title{
MODELING TO EVALUATE COORDINATION AND FLEXIBILITY IN ALUMINUM RECYCLING OPERATIONS
}

\author{
Tracey Brommer ${ }^{1}$, Elsa Olivetti ${ }^{1}$, Snorre Fjeldbo ${ }^{2}$, and Randolph Kirchain ${ }^{1}$ \\ ${ }^{1}$ Massachusetts Institute of Technology, \\ ${ }^{2}$ Hydro Aluminum
}

Key words: By product reuse, aluminum recycling, optimization and pooling

Reprocessing of aluminum production byproducts or dross for use in secondary production presents a particular challenge to the aluminum industry. While use of these non-traditional secondary materials is of interest due to their reduced energy and economic burden over virgin counterparts, these materials necessitate the use of particular furnaces, specialized handling and processing conditions. Therefore, to make use of them firms may pursue use of an intermediate recycling facility that can reprocess the secondary materials into a liquid product. After reprocessing downstream aluminum remelters could incorporate the liquid products into their aluminum alloy production schedules. Energy and environmental benefits result from delivering the products as liquid but coordination challenges result because of the energy cost to maintain the liquid. Further coordination challenges result from the need to establish long term recycling production plans in the presence of long term downstream aluminum remelter production uncertainty and inherent variation in the daily order schedule of the downstream aluminum remelters. In this context a fundamental question arises, considering the metallurgical complexities of dross reprocessing, what is the value of operating a coordinated set of by-product reprocessing plants and remelting cast houses?

A methodology is presented to calculate the optimal recycling center production parameters including the number and volume of intermediate products made in the recycling center, how these products should be allocated to downstream facilities given uncertainty in supply and demand. A long term production optimization model was used to evaluate the theoretical viability of the proposed two-stage scrap and aluminum dross reprocessing operation including the impact of reducing coordination on model performance. Then a dynamic simulation tool was used to evaluate the performance of the calculated recycling center production plan when resolved on a daily timeframe for varying levels of operational flexibility. The dynamic simulation revealed the optimal performance to correspond to the fixed recipe with flexible production daily optimization model formulation. Calculating recycled product specifications using the proposed simulation optimization method increased profitability when multiple downstream demand scenarios were included.

\section{Reference}

Brommer, T. "Recycling Production Designs: The Value of Coordination and Flexibility in Aluminum Recycling Operations." Ph.D. dissertation. Massachusetts Institute of Technology, Cambridge, MA. 2012. 